\title{
Weiterbildung \\ zwischen Grundrecht und Markt
}


Karin Derichs-Kunstmann

Peter Faulstich

Christiane Schiersmann

Rudolf Tippelt (Hrsg.)

\section{Weiterbildung zwischen Grundrecht und Markt}

Rahmenbedingungen und Perspektiven

Springer Fachmedien Wiesbaden GmbH 1997 
Die Deutsche Bibliothek - CIP-Einheitsaufnahme

Weiterbildung zwischen Grundrecht und Markt : Rahmenbedingungen und Perspektiven / Karin Derichs-Kunstmann ... (Hrsg.).

ISBN 978-3-8100-1646-1 ISBN 978-3-663-11824-4 (eBook)

DOI 10.1007/978-3-663-11824-4

NE: Derichs-Kunstmann, Karin

(C) 1997 Springer Fachmedien Wiesbaden

Ursprünglich erschienen bei Leske + Budrich, Opladen 1997

Das Werk einschließlich aller seiner Teile ist urheberrechtlich geschützt. Jede Verwertung außerhalb der engen Grenzen des Urheberrechtsgesetzes ist ohne Zustimmung des Verlages unzulässig und strafbar. Das gilt insbesondere für Vervielfältigungen, Übersetzungen, Mikroverfilmungen und die Einspeicherung und Verarbeitung in elektronischen Systemen. 


\section{Inhalt}

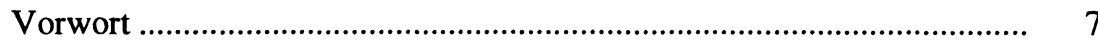

Einleitung

Peter Faulstich/Christiane Schiersmann/Rudolf Tippelt

Weiterbildung zwischen Grundrecht und Markt.

\section{Recht und Bildungspolitik}

\section{Gerhard Strunk}

Weiterbildung im Konflikt zwischen Grundrechtsverpflichtung und Marktanpassung. Von Ansätzen und Widersprüchen in

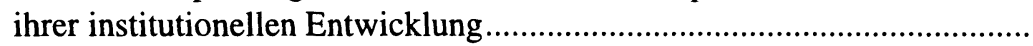

\section{Detlef Kuhlenkamp}

Regelungen und Realpolitik in der Weiterbildung

Klaus Künzel

Europäisierung der Weiterbildungspolitik

Markt und Systematisierung der Weiterbildung

Markt und Systematisierung der Weiterbildung

Ulrich Teichler

Politikprozesse, öffentliche Verantwortung und soziale Netzwerke..........

Peter Faulstich

Regulation der Weiterbildung - Markt, Staat und Netze

Christiane Schiersmann

Kooperation im regionalen Umfeld - Modelle und Erfahrungen 
Rudolf Tippelt

Neue Sozialstrukturen: Differenzierung von Weiterbildungsinteressen und Pluralisierung des Weiterbildungsmarktes

\section{Betriebliche Weiterbildung}

Rolf Dobischat

Weiterbildung im Kontext von Arbeitsmarktpolitik am Beispiel

der Reorganisation von beruflicher Weiterbildung im

Transformationsprozeß in den neuen Ländern.

Harald Geißler

Gemeinsamkeiten und Differenzen der institutionellen

Kontexte öffentlicher, privatwirtschaftlicher und

innerbetrieblicher Weiterbildung

Adressenliste der Autorinnen und Autoren 


\section{Vorwort}

Die Grundlage dieser Veröffentlichung bilden die Vorträge, die im Frühjahr 1996 auf dem Kongreß der Deutschen Gesellschaft für Erziehungswissenschaft in Halle im Rahmen eines vom Vorstand der Gesellschaft sowie der Kommission Erwachsenenbildung gemeinsam verantworteten Symposiums sowie der Arbeitsgruppe der Kommission Erwachsenenbildung gehalten wurden.

Wir freuen uns, für diese Publikation Herrn Budrich als Verleger gewonnen zu haben, und wir danken insbesondere Herrn Lars Wesener, M.A., für die intensive redaktionelle Bearbeitung des Manuskripts. 